PROCEEDINGS OF THE

AMERICAN MATHEMATICAL SOCIETY

Volume 137, Number 1, January 2009, Pages 247-253

S 0002-9939(08)09554-3

Article electronically published on August 15, 2008

\title{
REMARKS ON A FINSLER-LAPLACIAN
}

\author{
VINCENZO FERONE AND BERND KAWOHL
}

(Communicated by Walter Craig)

\begin{abstract}
We investigate elementary properties of a Finsler-Laplacian operator $Q$ that is associated with functionals containing $(H(\nabla u))^{2}$. Here $H$ is convex and homogeneous of degree 1 , and its polar $H^{o}$ represents a Finsler metric on $\mathbb{R}^{n}$. In particular we study the Dirichlet problem $-Q u=2 n$ on a ball $K^{o}=\left\{x \in \mathbb{R}^{n}: H^{o}(x)<1\right\}$ and present a fundamental solution for $Q$, suitable maximum and comparison principles, and a mean value property for solutions of $Q u=0$.
\end{abstract}

\section{Preliminaries}

Throughout this paper let $H: \mathbb{R}^{n} \mapsto \mathbb{R}$ be a nonnegative convex function of class $C^{2}\left(\mathbb{R}^{n} \backslash\{0\}\right)$ which is even and positively homogeneous of degree 1 , so that

$$
H(t \xi)=|t| H(\xi) \quad \text { for any } \quad t \in \mathbb{R}, \xi \in \mathbb{R}^{n} .
$$

A typical example is $H(\xi)=\left(\sum_{i}\left|\xi_{i}\right|^{q}\right)^{1 / q}$ for $q \in[1, \infty)$.

We shall investigate Euler equations which involve functionals containing the expression

$$
\int_{\Omega}(H(\nabla u))^{2} d x
$$

The differential equations contain operators of the form

$$
Q u:=\sum_{i=1}^{n} \frac{\partial}{\partial x_{i}}\left((H(\nabla u)) H_{\xi_{i}}(\nabla u)\right) .
$$

Note that these operators are not linear unless $H$ is the Euclidean norm. In particular, for $H(\xi)=\left(\sum_{k}\left|\xi_{k}\right|^{q}\right)^{1 / q}$ the operator $Q$ becomes

$$
Q u:=\sum_{i=1}^{n} \frac{\partial}{\partial x_{i}}\left(\left(\sum_{k=1}^{n}\left|\frac{\partial u}{\partial x_{k}}\right|^{q}\right)^{(2-q) / q}\left|\frac{\partial u}{\partial x_{i}}\right|^{q-2} \frac{\partial u}{\partial x_{i}}\right) \text {. }
$$

We set

$$
K:=\left\{x \in \mathbb{R}^{n}: H(x)<1\right\} .
$$

and $\omega_{n}=|K|$. Sometimes we will say that $H$ is the gauge of $K$. If one defines the support function of $K$ as

$$
H^{o}(x):=\sup _{\xi \in K}<x, \xi>,
$$

Received by the editors January 15, 2008.

2000 Mathematics Subject Classification. Primary 35J60, 53C60, 49Q20.

(C) 2008 American Mathematical Society Reverts to public domain 28 years from publication 
it is easy to verify that $H^{o}: \mathbb{R}^{n} \rightarrow[0,+\infty)$ is a convex, homogeneous function and that $H, H^{o}$ are polar to each other in the sense that

$$
H^{o}(x)=\sup _{\xi \neq 0} \frac{<x, \xi>}{H(\xi)}
$$

and

$$
H(x)=\sup _{\xi \neq 0} \frac{<x, \xi>}{H^{o}(\xi)} .
$$

For example, it follows that

$$
|<x, \xi>| \leq H(x) H^{o}(\xi) .
$$

Clearly $H^{o}(x)$ itself is the gauge of the set

$$
K^{o}:=\left\{x \in \mathbb{R}^{n}: H^{o}(x) \leq 1\right\} .
$$

We say that $K$ and $K^{o}$ are polar to each other. Finally we observe that

$$
H\left(\nabla H^{o}(x)\right)=1
$$

and, as a consequence of (1.1), that

$$
\sum_{i=1}^{n} H_{\xi_{i}}(\xi) \xi_{i}=H(\xi)
$$

\section{Constant datum}

The simplest case of the function $H(\xi)$ is given by $H(\xi)=|\xi|$, with $H^{o}(\xi)=|\xi|$. Obviously we have $Q u=\Delta u$, and it is not difficult to show that the function $u(x)=1-|x|^{2}$ solves the problem

$$
\begin{cases}-Q u=2 n & \text { in } B=\{x:|x|<1\} \\ u=0 & \text { on } \partial B\end{cases}
$$

We want to understand if a similar result holds true for a general operator $Q u$.

Example. Suppose that

$$
H(\xi)=\left(\sum_{k}\left|\xi_{k}\right|^{q}\right)^{1 / q}, \quad q>1 .
$$

Then it is easy to see that

$$
H^{o}(\xi)=\left(\sum_{k}\left|\xi_{k}\right|^{q^{\prime}}\right)^{1 / q^{\prime}}, \quad q^{\prime}=\frac{q}{q-1}
$$

and

$$
\frac{\partial}{\partial x_{i}}\left(\left(H^{o}(x)\right)^{2}\right)=2 H^{o}(x) \frac{\partial}{\partial x_{i}}\left(H^{o}(x)\right)=2\left(H^{o}(x)\right)^{2-q^{\prime}} x_{i}\left|x_{i}\right|^{q^{\prime}-2} .
$$

If $v(x)=\left(H^{o}(x)\right)^{2}$, then because of (1.7), we have for our example

$$
\begin{aligned}
Q v & =\sum_{i=1}^{n} \frac{\partial}{\partial x_{i}}\left(\left(H\left(\nabla\left(H^{o}(x)\right)^{2}\right)\right) H_{\xi_{i}}\left(\nabla\left(H^{o}(x)\right)^{2}\right)\right) \\
& =2 \sum_{i=1}^{n} \frac{\partial}{\partial x_{i}}\left(H^{o}(x) \frac{x_{i}}{H^{o}(x)}\right)=2 n .
\end{aligned}
$$


The calculation that we just did for the special $H$ in the example leads one to believe that the same result must hold for general $H$. We can in fact prove the following result in two very different ways.

Theorem 2.1. Consider the problem

$$
\begin{cases}-Q u=2 n & \text { in } K^{0} \\ u=0 & \text { on } \partial K^{0} .\end{cases}
$$

The solution to problem (2.3) is given by

$$
u(x)=1-\left(H^{o}(x)\right)^{2} .
$$

First proof. Follow the calculations leading up to (2.2) and observe that a) $H\left(\nabla H^{o}(x)\right)=1$, b) $H_{\xi_{i}}\left(\nabla\left(H^{o}(x)\right)^{2}\right)=H_{\xi_{i}}\left(\nabla H^{o}(x)\right)$, and finally c) $x=$ $H^{o}(x) H_{\xi}\left(\nabla H^{o}(x)\right)$. The last identity can be found in Lemma 2.2 of [4].

Second proof. The solution to problem (2.3) can be found by minimizing the functional

$$
J(u)=\int_{K^{0}}\left((H(\nabla v))^{2}-4 n v\right) d x .
$$

The minimizer $u$ of the functional $J$ on $H_{0}^{1}\left(K^{0}\right)$ is unique, and, because of the Pólya-Szegö inequality, it has to be such that

$$
u(x)=u\left(H^{o}(x)\right) .
$$

Indeed, if $u^{\#}$ denotes the convex symmetrization of $u$, then the following holds:

$$
J(u) \geq J\left(u^{\#}\right) .
$$

So we need only consider functions of the form

$$
v(x)=v\left(H^{o}(x)\right) .
$$

Taking into account (2.5) and (1.7) we have:

$$
J(u)=\int_{0}^{1} n \omega_{n}\left(\left(v^{\prime}(r)\right)^{2}-4 n v(r)\right) r^{n-1} d r
$$

The corresponding Euler equation of the one-dimensional problem is

$$
-\left(v^{\prime}(r) r^{n-1}\right)^{\prime}-2 n r^{n-1}=0 .
$$

We immediately have

$$
u(r)=1-r^{2},
$$

and then

$$
u(x)=1-\left(H^{o}(x)\right)^{2} .
$$

Remark 2.2. If $v(x)=\left(H^{o}(x)\right)^{2}$, then a straightforward calculation gives

$$
Q v=2\left(1+H^{o}(x) \sum_{i, j} H_{\xi_{i} \xi_{j}}\left(\nabla H^{o}(x)\right) H_{x_{i} x_{j}}^{o}(x)\right) .
$$

On the other hand, Theorem 2.1 implies $Q v=2 n$. Thus we have shown that

$$
H^{o}(x) \sum_{i, j} H_{\xi_{i} \xi_{j}}\left(\nabla H^{o}(x)\right) H_{x_{i} x_{j}}^{o}(x)=n-1,
$$

an identity which does not seem to be known. 
Remark 2.3. The case of nonconstant right-hand side $-Q u=f(u)$ was treated in [6], in particular the eigenvalue problem $f(u)=-\lambda u$. For $n=2$ and positive solutions we were able to show that all level sets of $u$ are homothetic to $K^{o}$, as in Theorem 2.1. Notice that Theorem 2.1 applies to general $n$ in the present paper. The desire to gain a deeper understanding of the behaviour of $Q$ for general $n$ was the motivation for our present study.

\section{Fundamental solution for the operator $Q$}

Our aim is to prove that when the datum of the Poisson equation involving the operator $Q$ is a Dirac delta, then as in Theorem 2.1 the solution can be written in terms of $H^{o}(x)$.

Theorem 3.1. The function

$$
u(x)= \begin{cases}\frac{1}{\alpha_{n}} \frac{\left(H^{o}(x)\right)^{-(n-2)}}{n-2} & \text { if } n>2, \\ -\frac{1}{\alpha_{2}} \log \left(H^{o}(x)\right) & \text { if } n=2\end{cases}
$$

solves, in the sense of distribution, the equation

$$
-Q u=\delta_{0}
$$

where $\alpha_{n}$ denotes the perimeter of the unit ball with respect to $H^{o}$ and $\delta_{0}$ denotes the Dirac measure at the origin.

Proof. By Theorem 2.1, a direct computation shows that

$$
-Q u(x)=0 \quad \text { for } x \neq 0 .
$$

For the benefit of the reader let us give the details of this calculation in dimension $n>2$. If we set $v(x)=\left(H^{o}(x)\right)^{2}$ and $w=\frac{2}{n-2} v^{-(n-2) / 2}$, we see that $\nabla w=$ $-v^{-n / 2} \nabla v$, so $H(\nabla w)=-v^{-n / 2} H(\nabla v)$. Since $H_{\xi}$ is homogeneous of degree zero, $H_{\xi}(\nabla w)=H-\xi(\nabla v)$. Thus, using Theorem 2.1,

$$
\begin{aligned}
-Q w & =\frac{\partial}{\partial x_{i}}\left(v^{-n / 2} H(\nabla v) H_{\xi_{i}}(\nabla v)\right) \\
& =v^{-n / 2} 2 n-\frac{n}{2} v^{-(n+2) / 2} H(\nabla v) H_{\xi_{i}}(\nabla v) \\
& =2 n v^{-(n+2) / 2}\left(v-\frac{1}{4} H^{2}(\nabla v)\right) .
\end{aligned}
$$

However, $H(\nabla v)=H\left(2 H^{o}(x) \nabla H^{o}(x)\right)=2 H^{o}(x)$, so that (3.3) holds.

In view of (3.3), in order to prove the theorem, it is sufficient to show that

$$
\lim _{\varepsilon \rightarrow 0} \int_{\partial K_{\varepsilon}^{0}} H(\nabla u) H_{\xi_{i}}(\nabla u) \nu_{i} \varphi d \sigma=\varphi(0), \quad \forall \varphi \in C_{0}^{\infty}\left(\mathbb{R}^{n}\right),
$$

where $K_{\varepsilon}^{0}=\left\{x \in \mathbb{R}^{n}: H^{o}(x) \leq \varepsilon\right\}$ and $\nu$ is the external normal to $\partial K_{\varepsilon}^{0}$. In our situation $\nu=-\nabla u / H(\nabla u)$. 
First of all we observe that $\partial K_{\varepsilon}^{0}$ is a level set for $u$ given in (3.1). Taking into account the homogeneity of the function $H$, we see this implies that

$$
\begin{aligned}
\int_{\partial K_{\varepsilon}^{0}} H(\nabla u) H_{\xi_{i}}(\nabla u) \nu_{i} \varphi d \sigma & =-\int_{\partial K_{\varepsilon}^{0}} H(\nabla u) H_{\xi_{i}}(\nabla u) \frac{u_{x_{i}}}{H(\nabla u)} \varphi d \sigma \\
& =-\int_{\partial K_{\varepsilon}^{0}} H(\nabla u) \varphi d \sigma .
\end{aligned}
$$

Now we observe that

$$
\nabla u=-\frac{1}{\alpha_{n}} \frac{\nabla H^{o}(x)}{\left(H^{o}(x)\right)^{n-1}},
$$

and, using again the homogeneity of $H$ and (1.7), we have

$$
H(\nabla u)=-\frac{1}{\alpha_{n}} H^{o}(x)^{1-n}=-\left(\alpha_{n} \varepsilon^{n-1}\right)^{-1},
$$

which proves the assertion for $n>2$. The proof for $n=2$ is left as an exercise for the reader.

Remark 3.2. It is worth noting that the fundamental solution does not in general give rise to a Poisson representation formula, because $Q$ is in general nonlinear. For the same reason we cannot construct Green's functions for Dirichlet problems on bounded domains.

\section{Maximum and COMPARISON PRINCIPLES FOR $Q$-HARMONIC FUnCTIONS}

We can easily prove the following weak maximum principle for $Q$-subharmonic functions.

Theorem 4.1. If $-Q u \leq 0$ in $\Omega$ and $u=g \leq M$ on $\partial \Omega$, then $u$ attains its maximum on the boundary; that is, $u(x) \leq M$ a.e. in $\Omega$.

Proof. We set $M=\max _{x \in \partial \Omega} g(x)$ and $\Omega^{+}:=\{x \in \Omega: u(x)>M\}$. Then we multiply $-Q u \leq 0$ by $(u-M)^{+}$and integrate over $\Omega^{+}$to obtain

$$
\begin{aligned}
0 & \geq \int_{\Omega_{+}} H(\nabla u) H_{\xi_{i}}(\nabla u) u_{x_{i}} d x+\int_{\partial \Omega^{+}} H(\nabla u)(u-M)^{+} H_{\xi_{i}}(\nabla u) \nu_{i} d \sigma(x) \\
(4.1) & =\int_{\Omega_{+}}(H(\nabla u))^{2} d x .
\end{aligned}
$$

Therefore $\Omega^{+}$has measure zero and $u(x) \leq M$ a.e. in $\Omega$.

In a similarly elegant way one can prove a comparison principle.

Theorem 4.2. Suppose $-Q u \leq-Q v$ in $\Omega$ and $u \leq v$ on $\partial \Omega$. Then $u \leq v$ a.e. in $\Omega$.

Proof. We assume that the set $\Omega^{+}:=\{u(x)>v(x)\}$ has positive measure and multiply the differential inequalities by $(u-v)^{+}$. Then

$$
\int_{\Omega^{+}}\left[H(\nabla u) H_{\xi}(\nabla u)-H(\nabla v) H_{\xi}(\nabla v)\right](\nabla u-\nabla v) d x \leq 0,
$$

so that by the strict convexity of $H^{2}$ we have $\nabla u=\nabla v$ a.e. in $\Omega^{+}$. Since $u=v$ on $\partial \Omega^{+}$we find that $\Omega^{+}$has measure zero. 


\section{Mean value Property For $Q$-Harmonic FunCtions}

Suppose $u$ satisfies $Q u=0$ in some domain $\Omega$ and $0 \in \Omega$. Then for sufficiently small $\rho$ the ball $K_{\rho}^{o}=\left\{x \in \mathbb{R}^{n}: H^{o}(x)<\rho\right\}$ is contained in $\Omega$. Harmonic functions are known to satisfy mean value properties. For $Q$-harmonic functions we can prove this only under the following assumption.

$$
<H_{\xi}(a), H_{\xi}^{o}(b)>=\frac{<a, b>}{H(a) H^{o}(b)} \quad \text { for all } a, b \in \mathbb{R}^{n} .
$$

This assumption is satisfied for

$$
H(\xi)=\left(\sum_{i}\left(\beta_{i}^{2} \xi_{i}^{2}\right)\right)^{1 / 2} \text { with } H^{o}(\eta)=\left(\sum_{i}\left(\eta_{i}^{2} / \beta_{i}^{2}\right)\right)^{1 / 2}
$$

but violated if $H(\xi)=\|\xi\|_{p}$ and $p \neq 2$.

Theorem 5.1. Suppose that $H$ and $H^{o}$ satisfy (5.1). If $Q u=0$ in $\Omega$ and $K_{\rho}^{o} \subset \Omega$, then for every ball of radius $r \in(0, \rho)$ the function $u$ satisfies the mean value property on spheres (where $\alpha_{n}$ denotes $\left|\partial K_{1}^{o}\right|$ ),

$$
u(0)=\frac{1}{\alpha_{n} r^{n-1}} \int_{\partial K_{r}^{o}} u(x) d \sigma
$$

as well as the corresponding mean value property on balls (where $k_{n}$ denotes $\left|K_{1}^{o}\right|$ ),

$$
u(0)=\frac{1}{k_{n} r^{n}} \int_{K_{r}^{o}} u(x) d x .
$$

Proof. We set

$$
\Phi(r):=\frac{1}{\alpha_{n} r^{n-1}} \int_{\partial K_{r}^{o}} u(x) d \sigma(x)=\frac{1}{\alpha_{n}} \int_{\partial K_{1}^{o}} u(r z) d \sigma(z)
$$

and show that $\Phi$ is in fact constant. Indeed,

$$
\Phi^{\prime}(r)=\frac{1}{\alpha_{n}} \int_{\partial K_{1}^{o}}<\nabla u(r z), z>d \sigma(z)=\frac{1}{\alpha_{n} r^{n-1}} \int_{\partial K_{r}^{o}}<\nabla u(x), \frac{x}{r}>d \sigma(x),
$$

and by (5.1) we have $\langle\nabla u, x\rangle=H(\nabla u)<H_{\xi}(\nabla u), H_{\xi}^{o}(x)>H^{o}(x)$. Therefore, since $H^{o}(x)=r$ and $\nu=H_{\xi}^{o}(x)$ on $\partial K_{r}^{o}$, an integration by parts yields

$$
\Phi^{\prime}(r)=\int_{\partial K_{r}^{o}} \sum_{i=1}^{n} H(\nabla u) H_{\xi_{i}}(\nabla u) \nu_{i} d \sigma(z)=\int_{K_{r}^{o}} Q u d \sigma(x)=0 .
$$

This proves the mean value property on spheres. The one for balls follows upon integration with respect to $r$.

Remark 5.2. Assumption (5.1) is not only sufficient but also necessary for the mean value property. Since $Q$ is translation-invariant, a counterexample can be constructed from the fundamental solution if one considers for instance $H(\xi)=\|\xi\|_{p}$ for $p$ close to $1, x_{0}=(2,0) \in \mathbb{R}^{2}$, and compares $u\left(x_{0}\right)$ to its mean value over the sphere $\partial K_{1}\left(x_{0}\right)$ of radius 1 centered at $x_{0}$.

Remark 5.3. Our notion of $Q$-harmonic function should not be confused with the "mean value Laplacian" from [7], 8] or the "Laplacian in Minkowski space" from 11. Contrary to our definition, Centore's and Thompson and Thompson's is linear in $u$. This discrepancy is a common phenomenon in Finsler geometry, where certain 
notions like volume, which have equivalent definitions in Euclidean space, provide different objects depending on the definition. Our implicit definitions of volume and perimeter follow the concept in [5].

\section{ACKNOWLEDGEMENTS}

This research was financially supported by GNAMPA. It is part of the ESF program "Global and Geometric Aspects of Nonlinear PDE". We thank G. Thorbergsson for several helpful discussions and him as well as M. Schott for bringing [1] to our attention.

\section{REFERENCES}

1. J.C. Álvarez Paiva and C. Durán, An Introduction to Finsler Geometry, Notas de la Escuela Venezolana de Matématicas, 1998.

2. J. C. Álvarez Paiva and A. C. Thompson, Volumes on normed and Finsler spaces (English summary). A sampler of Riemann-Finsler geometry, pp. 1-48, Math. Sci. Res. Inst. Publ. 50, Cambridge Univ. Press, Cambridge, 2004. MR.2132656 (2006c:53079)

3. A. Alvino, V. Ferone, G. Trombetti and P.-L. Lions, Convex symmetrization and applications. Ann. Inst. Henri Poincaré Anal. Non Linéaire 14 (1997) 275-293. MR1441395 (98d:35068)

4. G. Bellettini and M. Paolini, Anisotropic motion by mean curvature in the context of Finsler geometry. Hokkaido Math. J. 25 (1996) 537-566. MR.1416006 (97i:53079)

5. G. Bellettini, M. Paolini and S. Venturini, Some results on surface measures in calculus of variations. Ann. Mat. Pura Appl. (4) 170 (1996) 329-357. MR.1441625 (98e:49097)

6. M. Belloni, V. Ferone and B. Kawohl, Isoperimetric inequalities, Wulff shape and related questions for strongly nonlinear elliptic operators. J. Appl. Math. Phys. (ZAMP) 54 (2003), 771-783. MR2019179 (2005e:35177)

7. P. Centore, A mean-value Laplacian for Finsler spaces, in: The theory of Finslerian Laplacians and applications, pp. 151-186, Eds.: P.L. Antonelli and B.C. Lackey, Math. Appl., 459, Kluwer Acad. Publ., Dordrecht, 1998. MR1677362 (2000g:58051)

8. P. Centore, Finsler Laplacians and minimal-energy maps. Internat. J. Math. 11 (2000) 1-13. MR.1757888 (2001i:58067)

9. I. Fonseca and S. Müller, A uniqueness proof for the Wulff theorem. Proc. Royal Soc. Edinburgh 119 (1991) 125-136. MR1130601 (93c:49026)

10. R. D. Holmes and A. C. Thompson, $n$-dimensional area and content in Minkowski spaces. Pacific J. Math. 85 (1979) 77-110. MR571628 (81k:52023)

11. A. A. Thompson and A. C. Thompson, The divergence theorem and the Laplacian in Minkowski space. Geom. Dedicata 63 (1996) 159-170. MR1413628 (97j:52007)

Dipartimento di Matematica e Applicazioni "R. Caccioppoli", Università di Napoli "Federico II", Complesso Universitario Monte S. Angelo, Via Cintia, I-80126 Napoli, ITALY

E-mail address: ferone@unina.it

Mathematisches Institut, Universität Zu Köln, D-50923 Köln, Germany

E-mail address: kawohl@mi.uni-koeln.de 